\title{
Information Diversity in Coherent MIMO Radars
}

\author{
Salvatore Maresca \\ TeCIP Institute \\ Scuola Superiore Sant'Anna \\ Pisa, Italy \\ salvatore.maresca@santannapisa.it
}

\author{
Antonio Malacarne \\ PNTLab \\ CNIT \\ Pisa, Italy \\ antonio.malacarne@cnit.it
}

\author{
Paolo Ghelfi \\ PNTLab \\ CNIT \\ Pisa, Italy \\ paolo.ghelfi@cnit.it
}

\author{
Antonella Bogoni \\ PNTLAb \\ CNIT \\ Pisa, Italy \\ antonella.bogoni@cnit.it
}

\begin{abstract}
In this paper, the concept of information diversity in both the space and frequency domains is investigated for multiple-input multiple-output (MIMO) radars with widely separated antennas. Compared to phased-antenna arrays and multistatic radars, they can exploit more degrees of freedom, allowing them to maximize the information content upon centralized data fusion, thus granting unprecedented target detection and localization capabilities.

This analysis proceeds in parallel with the running progresses of microwave photonics (MWP), which could represent, in the near future, a new paradigm for the development of centralized MIMO radar architectures.

Thus, understanding the implications of information diversity becomes essential to foretell the system effectiveness in detecting and resolving closely spaced targets, as well as in suppressing sidelobes which may lead to false alarms. Performance metrics are proposed and evaluated to characterize the effects that information diversity has on centralized MIMO radars with widely separated antennas. On the other hand, the proposed methodology could reveal precious for designing the optimum system configuration.
\end{abstract}

Index Terms-MIMO Radar, Geometric Diversity, Frequency Diversity, Ambiguity Function, Coherence, Microwave Photonics

\section{INTRODUCTION}

Modern radar sensing applications continuously push for new system solutions with superior target detection and localization, as well as system stability and reliability features [1]. The increasing number and variety of possible scenarios, operative conditions, target characteristics and mission tasks (e.g., detection, tracking, classification, identification) has inevitably led the radar community to explore solutions based on data and/or information fusion paradigms [2].

Multistatic radar systems employ multiple closely and/or widely separated transmitting and receiving radar nodes [3]. When the antennas are widely separated, the system can exploit different target-sensor geometries for collecting a larger percentage of the power backscattered by the target, thus mitigating the problem of radar cross section (RCS) fluctuations, and for handling slow-moving targets.

However, multistatic radars typically rely on decentralized architectures, in which the remote radar nodes provide the central node, i.e., the one responsible for sensor fusion, only with pre-elaborated information (e.g., at the detection or

This work has been partially funded under the NATO Science for Peace and Security (SPS) SOLE Project, under the Grant no. G5267. track level). In other words, only a portion of the collected information is truly exploited.

On the contrary, centralized architectures ensure a better coordination among the network nodes, thus maximizing the exploitation of such information content. Multiple-input multiple-output (MIMO) radar systems, which belong to this category, have been studied for almost twenty years, proving to achieve superior performance by merging simultaneous observations from multiple sparse radar nodes [4]-[7]. As a matter of fact, enormous research efforts have always been directed towards a better understanding of such systems [7], as well as their performance in scenarios of interest [8], [9]. Unfortunately, until today the development of distributed MIMO radars has been hindered by two main problems: (i) the necessity of precise time/phase synchronization among the carrier signals at the radar nodes, and (ii) reliable bi-directional large-bandwidth long-range signal distribution among the remote nodes and the fusion centre.

As documented in [10]-[13], the hybrid discipline of microwave photonics (MWP), which bridges radio frequency (RF) applications to photonic processing techniques, has rapidly evolved to the point of attracting the interest of the radar communities. As a result, MWP techniques have been successfully applied in a number of fields: signal upand down-conversions with frequency flexibility and phase stability features [14], coherent multi-band operation [15], and coherent RF signal routing over optical fiber links [16], [17]. For these reasons, the increasing interest in photonic technology applied to microwave systems has opened new development perspectives, especially for MIMO radars. However, the potential benefits of MWP techniques applied to distributed MIMO radars have been only qualitatively investigated in [18].

This paper deals with the problem of information diversity in the spatial and frequency domains for MIMO radars with widely separated antennas. Relevant performance metrics are proposed to evaluate the system effectiveness in detecting and resolving closely spaced targets, as well as in suppressing sidelobes. Moreover, the proposed methodology represents a precious tool for designing the optimum system configuration.

The paper is organized as follows. Photonics-based MIMO radars are described in Section II. Information diversity, and its impact on resolution and sidelobes, together with some considerations on system non-idealities, is investigated in Section III. Finally, conclusions are drawn in Section IV. 


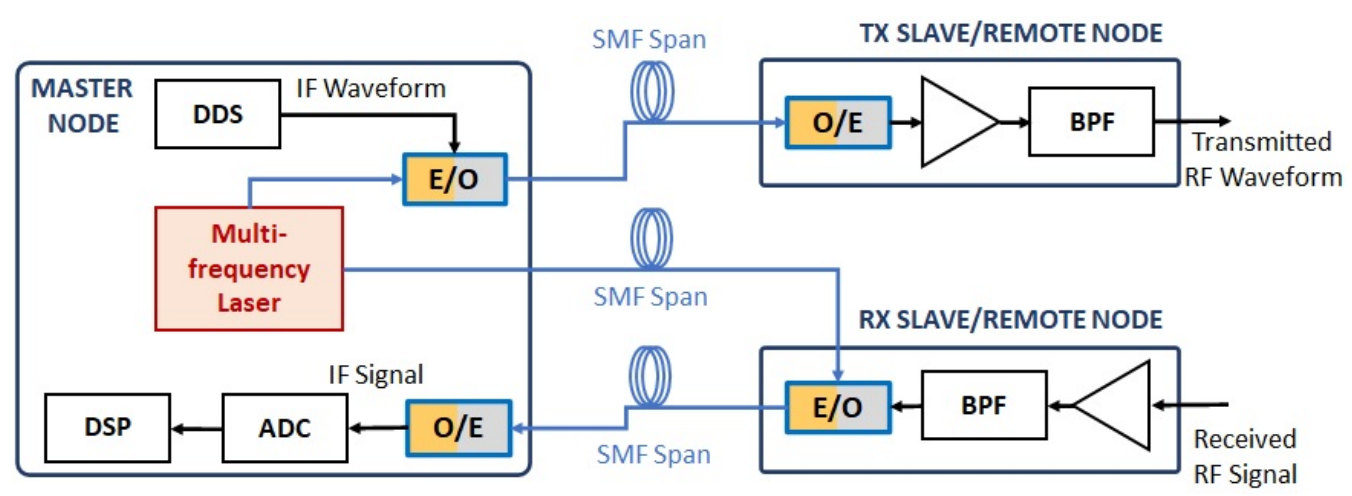

Fig. 1: Sample representation of a MIMO radar exploiting MWP techniques: multiple RF signal generation/reception and RF signal routing. E/O: electro-optical; O/E: opto-electrical; DDS: direct digital synthesizer; IF: intermediate frequency; ADC: analog to digital conversion; DSP: digital signal processing; SMF: single-mode fiber; BPF: band-pass filter.

\section{Photonics-BASEd MiMo Radars}

\section{A. MIMO Radar Architecture}

Let us consider a photonics-based distributed MIMO radar monitoring a given observation area, in which the pair $(x, y)$ denotes the cross-range and range coordinates of a generic point in the 2D Cartesian space. With no loss of generality, as shown in Fig. 1, the system consists of a master node, which contains the photonic core responsible of the RF signal generation and reception, the analog-to-digital conversion (ADC) and the digital signal processing (DSP) blocks. The system employs $M$ transmit (TX) and $N$ receive (RX) radar front-ends, which are not necessarily co-located. These are respectively characterized by the presence of an opto-electrical $(\mathrm{O} / \mathrm{E})$ and a electro-optical $(\mathrm{E} / \mathrm{O})$ conversion block. Both frontend types employ amplification and band-pass filter (BPF) stages for the selection of the operative RF carrier, respectively before and after the antennas. For easiness, the front-ends will be denoted with $T X_{m}$ and $R X_{n}$, being $m=1, \ldots, M$ and $n=1, \ldots, N$, with $M \neq N$.

Moreover, since MWP allows for software-defined coherent multi-band operation [15], [19], the photonic core can arbitrarily generate up to $L$ different waveforms at $L$ different carriers to be transmitted by $T X_{m}$. The system simultaneously illuminates $K$ point-like scattering elements, which can belong to a single target or to multiple targets.

\section{B. MIMO Radar Signal Model}

Being $s_{m, l}(t)$ the low-pass equivalent of the $l^{t h}$ signal transmitted by $T X_{m}$, with $l=1, \ldots, L$, the signal received by $R X_{n}$ can be written as in [6]:

$$
\begin{aligned}
& r_{m, n, l}(t)= \\
& \quad \sum_{k=1}^{K} a_{m, n, l}^{(k)} s_{m, l}\left(t-\tau_{m, n}^{(k)}\right) e^{j \varphi_{m, n}(t)}+w_{n}(t),
\end{aligned}
$$

where $a_{m, n, l}^{(k)}$ and $\tau_{m, n}^{(k)}$ are, respectively, the complex amplitude and delay characterizing the $k^{\text {th }}$ scattering element, with $k=1, \ldots, K$. These parameters are functions of the bistatic geometry between the scatterer location $P_{k}$ and the $T X_{m}$ and $R X_{n}$ sensor positions in the $2 \mathrm{D}$ Cartesian plane, as follows:

$$
\begin{gathered}
a_{m, n, l}^{(k)}=\sqrt{\frac{P_{T X}^{(m, l)} G_{T X}^{(m, l)} A_{n, l} \sigma_{m, n, l}^{(k)}}{(4 \pi)^{3} k_{B} B_{n, l} T_{n} L_{n} D_{1}^{2} D_{2}^{2}},} \\
\tau_{m, n}^{(k)}=\frac{1}{c}\left(D_{1}+D_{2}\right),
\end{gathered}
$$

where

$$
D_{1}=\mathbf{d}\left(T X_{m}, P_{k}\right), D_{2}=\mathbf{d}\left(P_{k}, R X_{n}\right) .
$$

Here, $P_{T X}^{(m, l)}$ and $G_{T X}^{(m, l)}$ are respectively the transmitted power and antenna gain at $T X_{m}$ for the $l^{\text {th }}$ waveform, $A_{n, l}$ is the effective area of the $R X_{n}$ antenna for the $l^{\text {th }} \mathrm{RF}$ carrier $f_{R F}^{(l)}, \sigma_{m, n, l}^{(k)}$ is the bistatic RCS of scatterer $P_{k}$ observed by $T X_{m}$ and $R X_{n}, k_{B}$ is the Boltzmann's constant, $c$ is the speed of light, $B_{n, l}$ is the noise bandwidth, $T_{n}$ and $L_{n}$ are the noise temperature and the loss factor at $R X_{n}$. Finally, $\mathbf{d}(A, B)$ is the Euclidean distance between two points $A$ and $B$.

Finally, the term $w_{n}(t)$ in eq. (1) is modelled as an additive white Gaussian noise (AWGN) stochastic process, while the term $\varphi_{m, n, l}(t)$ accounts for the overall phase shift introduced by the architecture. This drift is mainly caused by the optical oscillator instability and by the optical link [13]. The analysis carried out in [19] has investigated the impact of a $10 \mathrm{~km}$ single mode fiber (SMF) link on signal coherence, confirming its negligible effect on the short period, but highlighting a slight drift of about $5^{\circ}$ over multiple hours, mainly due to thermal fluctuations.

\section{MIMO Radar Processing}

Centralized processing is carried out at the master node, where the signals collected from all the RX front-ends are gathered and combined together at the DSP block. To estimate the target position, the maximum of the likelihood function of the target location $\vec{X} \equiv(x, y)$ must be evaluated from the set of all the $M \times N \times L$ available received signals $r_{m, n, l}(t)$. Thus, the target position can be estimated as the location $\vec{X}$ for which the log-likelihood function reaches its 
maximum [6]. This is the definition of the so-called MIMO ambiguity function $A(\vec{X})$. There are two ways to calculate it, as described in [4], [6], which depend on the aimed-for resolution, and, most importantly, on the available time/phase coherence among the radar channels.

As described in [4], if the angular jitter of the whole system is smaller than $0.1 \mathrm{rad}$, the phase error can be neglected. Thus, the following formula for the coherent MIMO ambiguity function can be used [4], [6]:

$$
A_{C}(\vec{X}) \propto\left|\sum_{m=1}^{M} \sum_{n=1}^{N} \sum_{l=1}^{L} e^{-j 2 \pi f_{R F}^{(l)} \tau_{m, n}} \Psi_{m, n, l}\left(t, \tau_{m, n}\right)\right|^{2},
$$

where:

$$
\Psi_{m, n, l}\left(t, \tau_{m, n}\right)=\int r_{m, n, l}^{*}(t) s_{m, l}\left(t-\tau_{m, n}\right) d t .
$$

For each possible target location $(x, y)$ in the Cartesian search space, the ambiguity function is computed determining the $M \times N \times L$ cross-correlations between the received and transmitted base-band equivalent signals, where ${ }^{*}$ indicates the operator of complex conjugation. The exponential terms $e^{-j 2 \pi f_{R F}^{(l)} \tau_{m, n}}$, which depend on the carrier frequency $f_{R F}^{(l)}$, are used to re-align the phases of the signals based on the travelled distance, proportional to the delays $\tau_{m, n}$. Thus, the complex correlation contributes can be summed together coherently. On the other hand, the non-coherent MIMO ambiguity function can be calculated neglecting the exponential terms in eq. (2) as follows [4], [6]:

$$
A_{N C}(\vec{X}) \propto\left|\sum_{m=1}^{M} \sum_{n=1}^{N} \sum_{l=1}^{L} \Psi_{m, n, l}\left(t, \tau_{m, n}\right)\right|^{2} .
$$

The non-coherent and coherent MIMO ambiguity functions, which are respectively described by eq. (7) and eq. (5), suggest two complementary functionalities of MIMO radar systems: the so-called "search" and "imaging" modes. For the interested reader, a detailed mathematical description can be found in [4]-[6]. The output of the search mode is typically used by a detection algorithm to reveal the possible presence of targets; once the targets have been spotted, the imaging mode can be applied to specific regions for target classification and recognition purposes.

Unfortunately, this latter operation is hindered by the overall angular jitter of the architecture. If this jitter remains below $10^{-1} \mathrm{rad}$ (i.e., $6^{\circ}$ ), its effects on MIMO coherent processing are negligible, as remarked in [4], [6]. For instance, the angular jitter of the system architecture described in [19] is in the order of $10^{-2} \mathrm{rad}$, far better than the limit identified in [6]. However, the phase drift introduced by the optical link, which could in principle affect the performance of the MIMO processing on the long term, must be periodically compensated.

Thus, MIMO radars based on photonics have the potential of providing different kinds of outputs and to carry out different mission tasks, such as target detection and imaging.

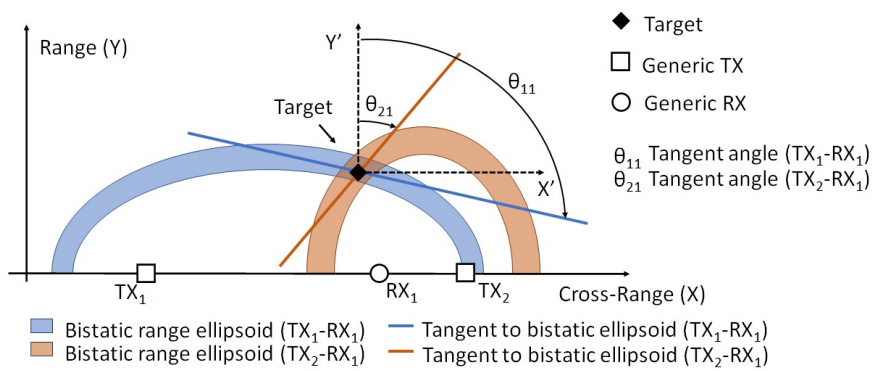

Fig. 2: Geometric Diversity for a MIMO radar employing 2 TX's and $1 \mathrm{RX}$ over a linear baseline.

\section{INFORMATION DIVERSITY}

Although still ongoing, this study is aimed at demonstrating the impact of a correct system dimensioning, in terms of sensor number and geometry, as well as waveform types and parameters, on MIMO radar performance. Different system configurations are simulated to demonstrate how the main issues, such as resolution and sidelobes, can be addressed by strategically profiting of geometric and frequency diversities.

\section{A. Geometric diversity}

As highlighted in [12], [19], a possible way to reduce the number and intensity of the undesired secondary lobes consists in taking advantage of the so-called geometric, or spatial, diversity gain. In order to quantify the degree of diversity achievable by a MIMO radar, a metric can be introduced, namely the geometric diversity parameter (GDP).

Given a sample MIMO array configuration as the one sketched in Fig. 2, the GDP corresponds to the standard deviation of the angles $\theta_{m, n}$, for $m=1, \ldots, M$ and $n=1, \ldots, N$, which are tangent to the bistatic ellipsoids generated by all the bistatic radar pairs $T X_{m}-R X_{n}$ at the target position:

$$
G D P=\operatorname{std}\left\{\theta_{m, n}\right\}_{m, n=1}^{M, N} .
$$

In other words, the larger is the GDP, the richer is the angular diversity provided by the MIMO radar channels. In fact, as shown in Fig. 2 such diversity has an effect on the area of intersection among the bistatic ellipsoids. In the ideal case in which the GDP is $90^{\circ}$, the cross-range resolution $\Delta X R$ for the non-coherent MIMO case of eq. (7) coincides with the range resolution $\Delta R$.

For a better understanding of the effect of geometric diversity, two sample MIMO radar configurations are considered, as depicted in Fig. 3. The two configurations, respectively indicated with A and B, are both composed by 2 TX's and 2 RX's placed along a $3 \mathrm{~m}$ fixed-length linear baseline. The GDP is calculated in a sample monitored space of $12 \times 12 \mathrm{~m}^{2}$. Configuration A exhibits a poor geometric diversity, see Fig. 4a, while configuration $\mathrm{B}$ is characterized by a better geometric diversity, see Fig. 4b.

The normalized MIMO ambiguity functions, see eq. (5), for the two configurations are calculated considering a point-like target placed at $3 \mathrm{~m}$ distance from the centre of the baseline. 


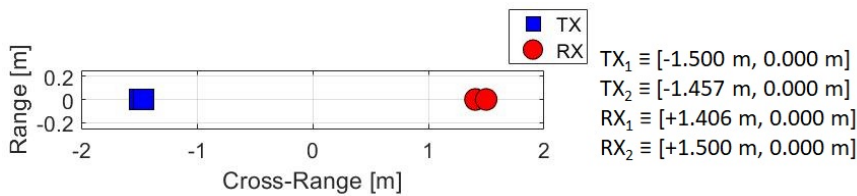

(a) Configuration $\mathrm{A}$

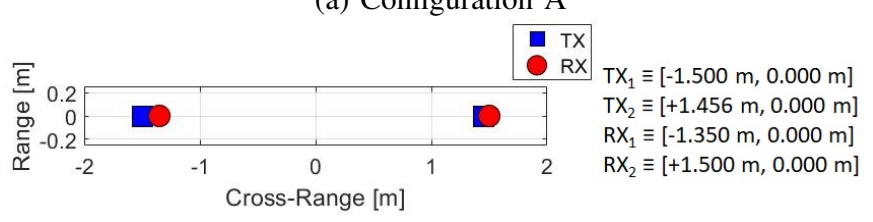

(b) Configuration $\mathrm{B}$

Fig. 3: Two sample $2 \times 2$ MIMO radar configurations with TX's and RX's distributed along a fixed $3 \mathrm{~m}$ baseline: (a) Configuration A with poor geometric diversity, (b) Configuration B with good geometric diversity.

At this target range, we observe $\mathrm{GDP}_{A} \approx 0^{\circ}$, while $\mathrm{GDP}_{B}=$ $20^{\circ} \div 25^{\circ}$, respectively for configurations $\mathrm{A}$ and $\mathrm{B}$.

For the simulations, an indoor scenario, similar to the one proposed [17], is considered. Here, the transmitted waveforms are linear frequency modulated continuous wave (LFMCW) signals, with carrier frequency $f_{R F}=10 \mathrm{GHz}$, bandwidth $B=1 \mathrm{GHz}$, and duration $T_{p}=275$ ns. Upchirp and down-chirp waveforms are transmitted by $T X_{1}$ and $T X_{2}$, respectively. Results are depicted in Fig. 5. As we can observe in Fig. 5a, a poor geometric diversity may prevent the system from resolving the target in cross-range, thus making the coherent system behave as in the non-coherent case. Instead, when the MIMO array configuration exhibits a good geometric diversity, the resolution capabilities of the system improve accordingly, as shown in Fig. 5b. However, due to the limited number of antenna elements, many secondary lobes appear around the mainlobe. These lobes could represent a serious problem leading to false alarms in the detection phase.

It is worth noticing that, for any MIMO radar configuration, the GDP tends to decrease as the target range increases. This means that, with increasing distance, the virtual channels become more spatially correlated [6], as shown in Fig. 4b in the case of configuration $\mathrm{B}$. This fact may dramatically increase the possibility of having high sidelobes in the coherent MIMO ambiguity function. A possible solution is to increase the number of antenna elements. In this paper, the effect of geometric diversity on the system detection and resolution capabilities is only qualitatively introduced.

\section{B. Frequency diversity}

In MIMO radars, system performance depends not only on the sensor-target relative geometry, but also on the waveform parameters. When signals on multiple coherent bands are transmitted, these latter may provide not only additional information about the target scattering characteristics, but also contribute to an overall improvement of the system resolution capabilities.

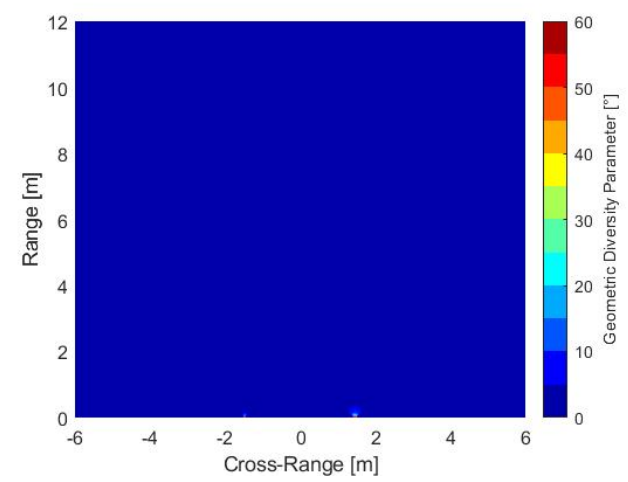

(a) Configuration $\mathrm{A}$

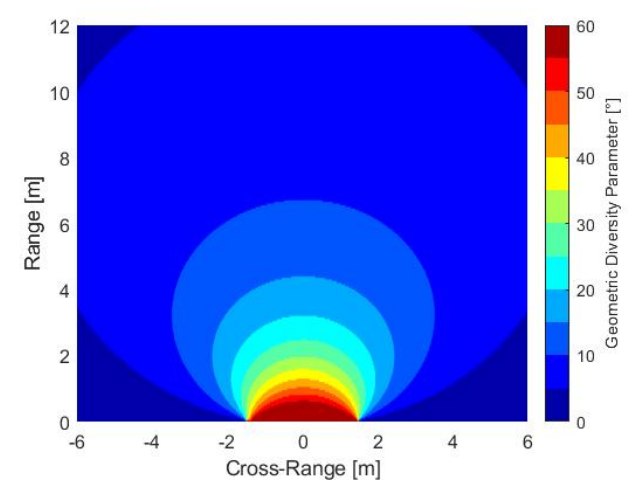

(b) Configuration $\mathrm{B}$

Fig. 4: Geometric diversity parameters (GDPs) evaluated for the two 2x2 MIMO radar configurations depicted in Fig. 3: (a) Configuration A with poor geometric diversity, (b) Configuration B with good geometric diversity.

This improvement is qualitatively shown in Fig. 6 for the two configurations described in Section III-A. Simulations are carried out considering a coherent dual-band operation, with $f_{\mathrm{RF}}^{(1)}=7 \mathrm{GHz}$ and $f_{\mathrm{RF}}^{(2)}=10 \mathrm{GHz}$, and $B_{1}=B_{2}=1 \mathrm{GHz}$. The estimated values of range and cross-range resolutions, respectively denoted with $\Delta R$ and $\Delta X R$, measured from the peak width at $-3 \mathrm{~dB}$ are reported in Table I.

The cross-range resolution in the case of configuration $\mathrm{A}$ is very coarse, and worsens in the case of dual-band operation, i.e., from 61 to $73 \mathrm{~cm}$. Conversely, the cross-range resolution in both the single- and dual-band cases of configuration B is approximately $1 \mathrm{~cm}$. This value is perfectly aligned with the theoretical limit given by the ratio between the carrier wavelength and the baseline length. Moreover, the dual-band operation in the case of configuration B, see Fig. 6b, allows to mitigate up to a certain extent the secondary lobes present in the single-band case, see Fig. 5b.

In addition to this, by comparing the outputs of Fig. 5a with Fig. 6a, and the outputs of Fig. 5b with Fig. 6b, it is possible to observe an interesting improvement of range resolution, too. In fact, in the single-band cases, see Fig. 5, the range resolution corresponds to the nominal resolution achieved by the waveform bandwidth, i.e., $15 \mathrm{~cm}$. This resolution 


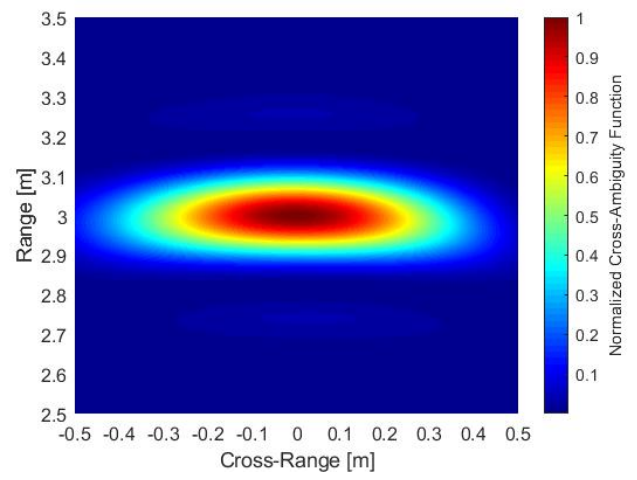

(a) Configuration $\mathrm{A}$

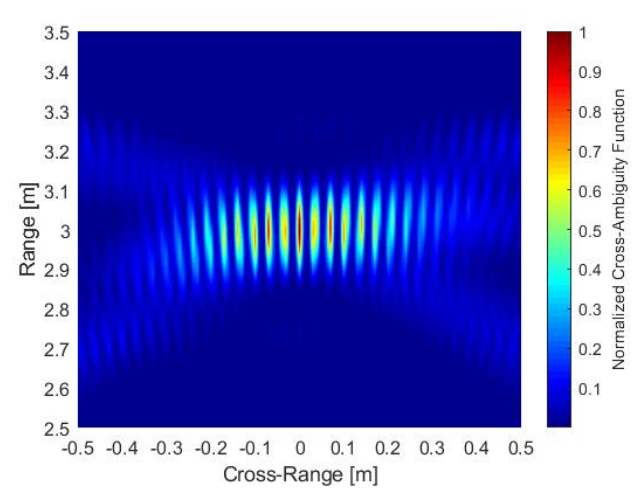

(b) Configuration $\mathrm{B}$

Fig. 5: Coherent single-band MIMO outputs, for the two $2 \times 2$ MIMO radar configurations of Fig. 3: (a) Conf. A with poor geometric diversity, (b) Conf. B with good geometric diversity.

improves to 4 and $3 \mathrm{~cm}$ in the dual-band cases, respectively for configurations A and B, see Fig. 6. However, together with this improvement, secondary "vestigial" lobes appear along range.

As depicted in Fig. 7, it is worth to be noted that the coherent dual-band case (red curve) consists in a sort of beating (interfering) pattern that is enveloped by the result of the single $1 \mathrm{GHz}$-band case (blue curve). As the results from each of the two bands, with central frequencies of $7 \mathrm{GHz}$ and $10 \mathrm{GHz}$ respectively, are coherently summed (in amplitude and phase), the resulting "pulse" consists in a pattern, whose complex field beats (interferes) at the difference of the two carrier frequencies (i.e., at $3 \mathrm{GHz}$ ), where the intensity one interferes at double frequency, i.e., at $6 \mathrm{GHz}$.

\begin{tabular}{|c|c|c|c|c|}
\hline & Fig. 5a & Fig. 5b & Fig. 6a & Fig. 6b \\
\hline$\Delta R$ & $0.15 \mathrm{~m}$ & $0.15 \mathrm{~m}$ & $0.04 \mathrm{~m}$ & $0.03 \mathrm{~m}$ \\
\hline$\Delta X R$ & $0.61 \mathrm{~m}$ & $0.01 \mathrm{~m}$ & $0.73 \mathrm{~m}$ & $0.01 \mathrm{~m}$ \\
\hline
\end{tabular}

TABLE I: Resolution estimates in range $(\Delta R)$ and cross-range $(\Delta X R)$ for the coherent MIMO outputs of Figs. 5, 6.

\section{Analysis of Sidelobes}

The coherent MIMO ambiguity function, see eq. (5), can be considered equivalent to a beamforming operation applied to a sparse array of antennas. Unfortunately, since contributions

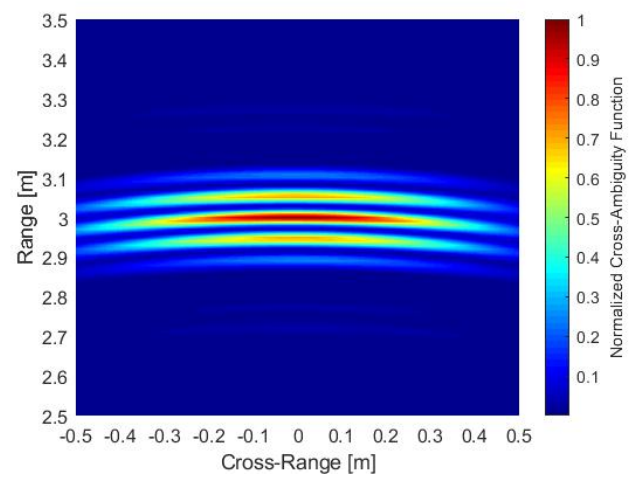

(a) Configuration $\mathrm{A}$

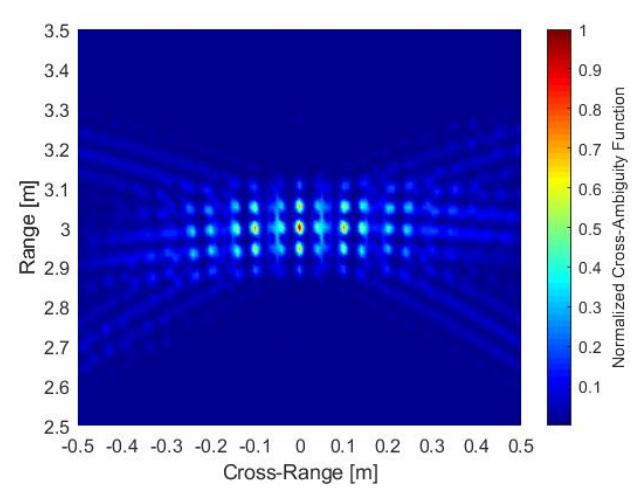

(b) Configuration $\mathrm{B}$

Fig. 6: Coherent dual-band MIMO outputs, for the two $2 \times 2$ MIMO radar configurations of Fig. 3: (a) Conf. A with poor geometric diversity, (b) Conf. B with good geometric diversity.

from different frequency ranges are summed in both amplitude and phase, it is not straightforward to predict the impact of both the frequency bands and the baseline geometry, on both resolution and on sidelobes disposition and contribution.

For addressing this problem, two metrics can be defined for quantifying the maximum and average intensity level of the sidelobes with respect to the mainlobe. These metrics are the peak-to-maximum sidelobe ratio (PMSR) and peak-to-average sidelobe ratio (PASR), which are respectively calculated as:

$$
\begin{aligned}
& P M S R=\max _{\bar{k} \in K}\left\{A_{C}^{(\bar{k})}\right\} / \max _{k \in K \backslash \bar{k}}\left\{A_{C}^{(k)}\right\}, \\
& P A S R=\max _{\bar{k} \in K}\left\{A_{C}^{(\bar{k})}\right\} / \operatorname{mean}_{k \in K \backslash \bar{k}}\left\{A_{C}^{(k)}\right\},
\end{aligned}
$$

where $A_{C}^{(k)}$ is the value of the coherent ambiguity function at the $k^{t h}$ local maximum, $\bar{k}$ is the index of the absolute maximum corresponding to the position of the target, while $K$ represents the total number of local maxima.

The PMSR and the PASR have been calculated over 1000 Monte Carlo runs, in which the TX and RX antennas have been randomly placed along the same $3 \mathrm{~m}$ baseline. In all simulations, a point-like target placed at $3 \mathrm{~m}$ along range has been considered. The average values reported in Table II confirm the impact that the number of antennas and their position have on the system capability to suppress sidelobes. 
Moreover, as described in III-B, the use of multiple, strategically chosen, carriers further improves the sidelobe suppression capability of the system in terms of both peak and average sidelobe ratios. A detailed study that links the information diversity parameters with the key performance metrics is still object of ongoing investigations.

\begin{tabular}{|c|c|c|c|c|}
\hline & $2 \times 2 \mathbf{S B}$ & $2 \times 2 \mathbf{D B}$ & $4 \times 4 \mathbf{S B}$ & $4 \times 4 \mathrm{DB}$ \\
\hline$\overline{P M S R}$ & $0.85 \mathrm{~dB}$ & $2.97 \mathrm{~dB}$ & $4.89 \mathrm{~dB}$ & $7.78 \mathrm{~dB}$ \\
\hline$\overline{P A S R}$ & $16.61 \mathrm{~dB}$ & $18.28 \mathrm{~dB}$ & $22.16 \mathrm{~dB}$ & $24.22 \mathrm{~dB}$ \\
\hline
\end{tabular}

TABLE II: Average values of the peak-to-maximum sidelobe ratio (PMSR) and peak-to-average sidelobe ratio (PASR) for the configurations in Fig. 7. SB: Single-Band; DB: Dual-Band.

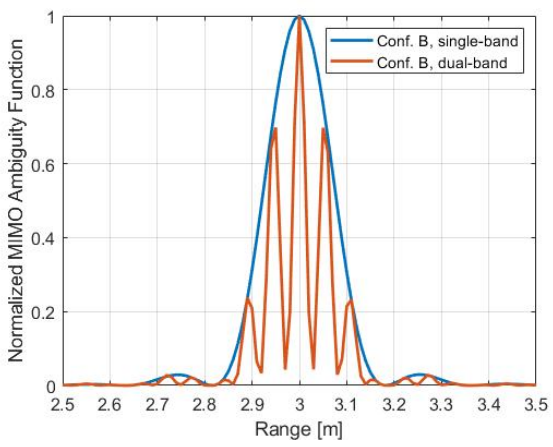

Fig. 7: Normalized coherent ambiguity function along range for the $2 \times 2$ MIMO radar Configuration B: single-band operation (blue curve); dual-band operation (red curve).

\section{System Non-Idealities}

To understand up to which extent the results from real and simulated data can be considered similar, it is convenient to summarize the findings of [17], where a MIMO radar-overfiber (Raof) system has been proposed, based on vertical cavity surface emitting lasers (VCSELs). The comparison under the same operative conditions between experimental and simulated results has demonstrated that the position and the amplitude of the main and secondary lobes in the coherent ambiguity function are almost identical.

To the best of our knowledge, the main differences between the two types of results could be ascribed to: (i) the scattering nature of real targets, which are not point-like scatterers, but rather distributed targets; (ii) the unpredictable multipath scattering features of the background in the experiment (i.e., indoor); (iii) the temporal granularity given by the ADC sampling rate (e.g., a $40 \mathrm{GS} / \mathrm{s}$ rate leads to a phase alignment accuracy of about $2 \pi / 5$ ); (iv) the unpredictable temperaturedependent variations of the VCSEL gain which, if not compensated, may lead to possible amplitude mismatches between the channels. All these issues may negatively affect the position of the main lobe and lead to increased sidelobes.

\section{CONCLUSION}

In this paper, the concept of information diversity has been investigated for coherent MIMO radars. Since it is no easy task to understand the dependency of the coherent MIMO ambiguity function from sensor geometry and waveform parameters, a preliminary analysis has been proposed, in which key performance metrics have been defined to link the system sidelobe suppression and target resolution capabilities to geometric and frequency diversity information.

This analysis, which does not mean to be exhaustive, has started answering the very first question: the strength of coherent MIMO radars dwells in the multi-perspective nature of the collected information. In fact, assuming signal coherence, as demonstrated in other real-world applications of MWP techniques, it has been shown that $2 \mathrm{D}$ resolution is determined by the baseline dimension and signal frequency, whereas the number of sensors is fundamental for reducing the amplitude of the harmful sidelobes. Moreover, implementing multi-band systems effectively reduces the sidelobes without further increasing the sensor number, thanks to the decorrelated position of sidelobes given by different signal frequencies.

\section{REFERENCES}

[1] M. S. Greco, J. Li, T. Long, and A. Zoubir, "Advances in Radar Systems for Modern Civilian and Commercial Applications: Part 1 [From the Guest Editors]," IEEE Sig. Proc. Mag., vol. 36, no. 4, pp. 13 - 15 , July 2019.

[2] D. L. Hall, and J. Llinas, "An introduction to multisensor data fusion," Proc. of the IEEE, vol. 85, no. 1, pp. 6-23, 1988.

[3] V. S. Chernyak, Fundamentals of Multisite Radar System, Gordon \& Breach Science Publ., London, 1998.

[4] N. H. Lehmann, et al., "High resolution capabilities of MIMO radar," Proc. of the $40^{\text {th }}$ Asilomar Conf. on Sig., Sys., and Comp., Nov. 2006.

[5] J. Li and P. Stoica, "MIMO Radar with Colocated Antennas," IEEE Sig. Proc. Mag., vol. 24, no. 5, pp. 106 - 114, Sept. 2007.

[6] A. M. Haimovich, et al., "MIMO Radar with Widely Separated Antennas," IEEE Sig. Proc. Mag., vol. 116, 2008.

[7] J. Li, and P. Stoica, MIMO Radar Signal Processing, John Wiley \& Sons Inc., 2008.

[8] N. H. Lehmann, et al., "MIMO-radar application to moving target detection in homogeneous clutter," Adaptive Sensor Array Processing Workshop at MIT Lincoln Laboratory, Waltham, MA, July 2006.

[9] A. A. Gorji, et al., "Widely Separated MIMO versus Multistatic Radars for Target Localization and Tracking," IEEE Trans. on Aerosp. and Electr. Sys., vol. 49, no. 4, pp. 2179 - 2194, Oct. 2013.

[10] J. Capmany and D. Novak, "Microwave photonics combines two worlds," Nature Photonics, vol. 1, pp. 319 - 330, 2007.

[11] J. McKinney, "Photonics illuminates the future of radar," Nature, vol. 507, pp. 310-312, 2014.

[12] A. Bogoni, P. Ghelfi, and F. Laghezza, Photonics for Radar Networks and Electronic Warfare Systems, IET SciTech Pub., London, 2019.

[13] G. Serafino, et al., "Microwave Photonics for Remote Sensing: From Basic Concepts to High-Level Functionalities," IEEE J. of Light. Tech., vol. 38 , no. 19 , pp. $5339-5355$, Oct. 2020.

[14] G. Serafino et al., "Phase and Amplitude Stability of EHF-Band Radar Carriers Generated From an Active Mode-Locked Laser," IEEE J. of Light. Tech., vol. 29, no. 23, pp. 3551 - 3559, Dec. 2011.

[15] P. Ghelfi, et al., "Photonics for Radars Operating on Multiple Coherent Bands," IEEE J. of Light. Tech., vol. 34, no. 2, pp. 500-507, Jan. 2016.

[16] G. Serafino, et al., "Toward a New Generation of Radar Systems Based on Microwave Photonic Technologies," IEEE J. of Light. Tech., Vol. 37, n. 2 , pp. $643-650,2019$.

[17] A. Malacarne, et al., "Coherent Dual-Band Radar-Over-Fiber Network With VCSEL-Based Signal Distribution," IEEE J. of Light. Tech., vol. 38 , no. 22 , pp. $6257-6264$, Nov. 2020.

[18] L. Lembo, et al, "Analysis of a Coherent Distributed MIMO PhotonicsBased Radar Network," Proc. of the 2018 Europ. Radar Conf. (EuRAD), Sept. 2018

[19] F. Scotti, et al., "Widely Distributed Photonics-Based Dual-Band MIMO Radar for Harbour Surveillance," IEEE Phot. Tech. Lett., vol. 32, no. 17, pp. $1081-1084$, Sept. 2020. 